\title{
Plasma Concentration of Osteopontin (OPN) in Children with Systemic Lupus Erythematosus: Relationship with Disease Activity
}

\author{
Mohamed Farouk Afify ${ }^{1}$, Gamal B. Mohamed ${ }^{*},{ }^{1}$, Mohamed Abd El-Maboud $^{1}$ and \\ Esmat A. Abdel-Latif ${ }^{2}$ \\ ${ }^{1}$ Departments of Pediatrics, and ${ }^{2}$ Clinical Pathology, Faculty of Medicine, Al-Minya University, Egypt
}

\begin{abstract}
Background: Osteopontin (OPN) is an important bone matrix mediator found to have key roles in inflammation and immunity. OPN is a cytokine which can play a number of roles in promoting activation of $\mathrm{T}$ lymphocyte, regulating balance between T-helper 1 and T-helper 2, participating in cell-induced immunologic response and stimulating B lymphocyte to express multi-clone antibodies. Overexpression of OPN has been associated with the development of the autoimmune/ lymphoproliferative syndrome.
\end{abstract}

Objective: The aim of our present study was to analyze the possible correlation between the plasma concentration of OPN and disease activity in children with systemic lupus erythematosus (SLE). We also investigated the correlation between plasma IL-18 and OPN concentrations to further confirm the association of OPN with disease activity.

Methods: We measured the plasma concentration of OPN, and the plasma proinflammatory IL-18 concentration in 40 SLE patients with or without renal disease (RSLE group and SLE group, respectively) and in 30 sex- and age-matched controls using enzyme immunoassay.

Results: Plasma OPN concentrations were significantly higher in RSLE and SLE patients than in the controls $(p=0.000$ and $\mathrm{p}=0.002)$. Plasma OPN concentrations were significantly higher in RSLE patients than in the SLE patients ( $\mathrm{p}=$ 0.000). Plasma OPN concentration correlated positively and significantly with SLE disease activity index in combined SLE patient groups $(\mathrm{r}=0.34 ; \mathrm{p}=0.04)$. In RSLE patients, plasma OPN concentration showed a significant positive correlation with proinflammatory cytokine IL-18 concentration $(r=0.48 ; p=0.004)$.

Conclusion: The above results suggest that the production of OPN is associated with the inflammatory process and SLE development, and may serve as a potential disease marker of SLE.

Keywords: SLE, Osteopontin, IL-18, SLEDAI, Children.

\section{INTRODUCTION}

Systemic lupus erythematosus (SLE) is a disorder of immune regulation manifested by the activation of $\mathrm{T}$ lymphocytes and polyclonal B lymphocytes, the production of autoantibodies and the formation of immune complexes causing tissue damage. Lupus nephritis is a frequent and potentially serious complication of SLE $[1,2]$. The aetiology and pathogenic mechanism of this immunological disorder have not been clearly elucidated. Complex networks of cytokines have been shown to regulate $\mathrm{T}$-and B-cell growth, differentiation and effector functions. They can be grouped into the cyokines that are associated with T-helper cells of type 1 (Th1) [e.g. interleukin (IL)-2, IL-12, IL-18, interferon- $\gamma$ (IFN- $\gamma$ ) and tumour necrosis factor- $\alpha(\mathrm{TNF}-\alpha)]$, which induce cell-mediated immunity, and those that are associated with T-helper cells of type 2 (Th2) (e.g. IL-4 and IL-10), which stimulate antibody production $[3,4]$. Previous findings demonstrated that the plasma concentration of proinflammatory cytokine IL-18 was significantly elevated in SLE patients compared with controls [4]. Studies of Tokano et al.

*Address correspondence to this author at the Departments of Pediatrics, Faculty of Medicine, Al-Minya University, Egypt;

E-mail: gamalbaheeg@yahoo.com
[5] and of Wong et al. [6] showed that plasma IL-12 concentrations in SLE patients were significantly higher than those of normal subjects. The combination of IL-12 and IL-18 is critical for the induction of the innate immune response and inflammatory reaction in SLE [7]. Wong et al. [6] found a positive correlation between the plasma IL-18/IL-4 ratio and SLE disease activity index (SLEDAI), suggesting an imbalanced cytokine profile with Th1 predominance. Akahoshi et al. [8] also reported that the ratio of intracellular IFN- $\gamma / \mathrm{IL}-4$ staining of Th cells did not support a predominance of Th2 in SLE. In contrast, they found that there was a significant Th1 predominance among SLE patients with WHO class IV lupus nephritis and diffuse proliferative glomerulonephritis.

Osteopontin (OPN) is a secreted phosphorylated glycoprotein expressed in mineralized tissues (bone and teeth) and damaged renal tissues. During inflammation, OPN is expressed by macrophage and T lymphocyte. OPN plays a number of roles in promoting activation of $\mathrm{T}$ lymphocyte, affecting the differentiation of $\mathrm{T}$ lymphocyte into Th1 and Th2 type cell, regulating the balance between Th1 and Th2, and participating in the cell-induced immunologic response. At the same time, OPN can stimulate B lymphocyte to express multi-clone antibodies [9-12]. The in vitro production of OPN is modulated by IL-2, transforming growth factor- $\beta$, epidermal growth factor and platelet-derived growth factor 
[11]. Among multiple receptors for OPN, CD44 is the most characterized receptor that appears to mediate the cell chemotaxis and attachment $[13,14]$. OPN plays various biological roles for host defence, bone formation, osteoclast activation and wound healing [15]. Its cytokine activities include the stimulation of macrophage and T-cell migration $[12,16]$, protection against herpes viruses and bacterial infections through the activation of the Th1 response, and induction of Th1-cell-mediated autoimmunity [12].

Increased plasma concentration, protein expression and local production of OPN have been observed in sepsis [17], metastasis [18], multiple sclerosis [19], autoimmune/lympho-proliferative syndrome [20], renal tissue of SLE patients [21], and rheumatoid joints of patients with rheumatoid arthritis [22]. However, the circulating level of OPN in SLE patients and its correlation with disease severity has not been well defined. Therefore, the aim of our present study was to estimate plasma concentrations of OPN and IL18 in children with SLE, and to analyze the possible correlation between the plasma concentration of OPN and disease activity in children with SLE with or without renal disease. We also investigated the correlation between plasma IL-18 and OPN concentrations to further confirm the association of OPN with disease activity.

\section{SUBJECTS AND METHODS}

Forty SLE patients were recruited from the Pediatric Outpatient Clinic of Suzan Moubarak Hospital of Al-Minya University and from the Rheumatology Out-patient Clinic of Al-Minya University Hospital over a period of 2 years from the first of August 2006 to the end of July 2008. Children were considered eligible for inclusion in the analysis if they fulfilled the following criteria: (1) American College of Rheumatology (ACR) criteria for the diagnosis of SLE [23]; (2) age $\leq 16$ years at the time of diagnosis of SLE. Disease activity of SLE was evaluated with the SLEDAI [24]. The
SLE patients were divided into two groups: 20 SLE patients with renal disease characterized by continuous proteinuria over $0.5 \mathrm{~g} / 24 \mathrm{~h}$ [23] (RSLE group) and 20 SLE patients without renal disease (SLE group). The study included as well 30 age- and sex- matched healthy children comprised the control group. Five $\mathrm{ml}$ of venous peripheral blood was collected from each patient and control subject. Informed consent was obtained from all participants. Concentrations of OPN and IL-18 in plasma were measured by enzyme immunoassay using reagent kits from Assay Designs (MI, USA) and BioSource (CA, USA) respectively.

\section{STATISTICAL METHODS}

After collection of data, they were added and entered into a personal computer. Analysis of the data was done using SPSS, version 13. The following statistical tests were used:

1. Mean and standard deviation (SD) to describe quantitative data.

2. Student $\mathrm{t}$ test was used to compare between two groups as regards parametric data.

3. Pearson correlation was used to correlate two quantitative variables.

For all tests, a probability (p) of less than 0.05 was considered significant.

\section{RESULTS}

\section{SLE Patients and Control Subjects}

The sex, age, duration of diagnosis, SLEDAI score and drug treatment of the study groups are summarized in Table 1. The disease activity of SLE patients was evaluated by their clinical parameters; the proportions of the RSLE group and SLE group with active disease were $85 \%$ and $45 \%$, respectively.

Table 1. Characteristics of RSLE Patients, SLE Patients and Controls

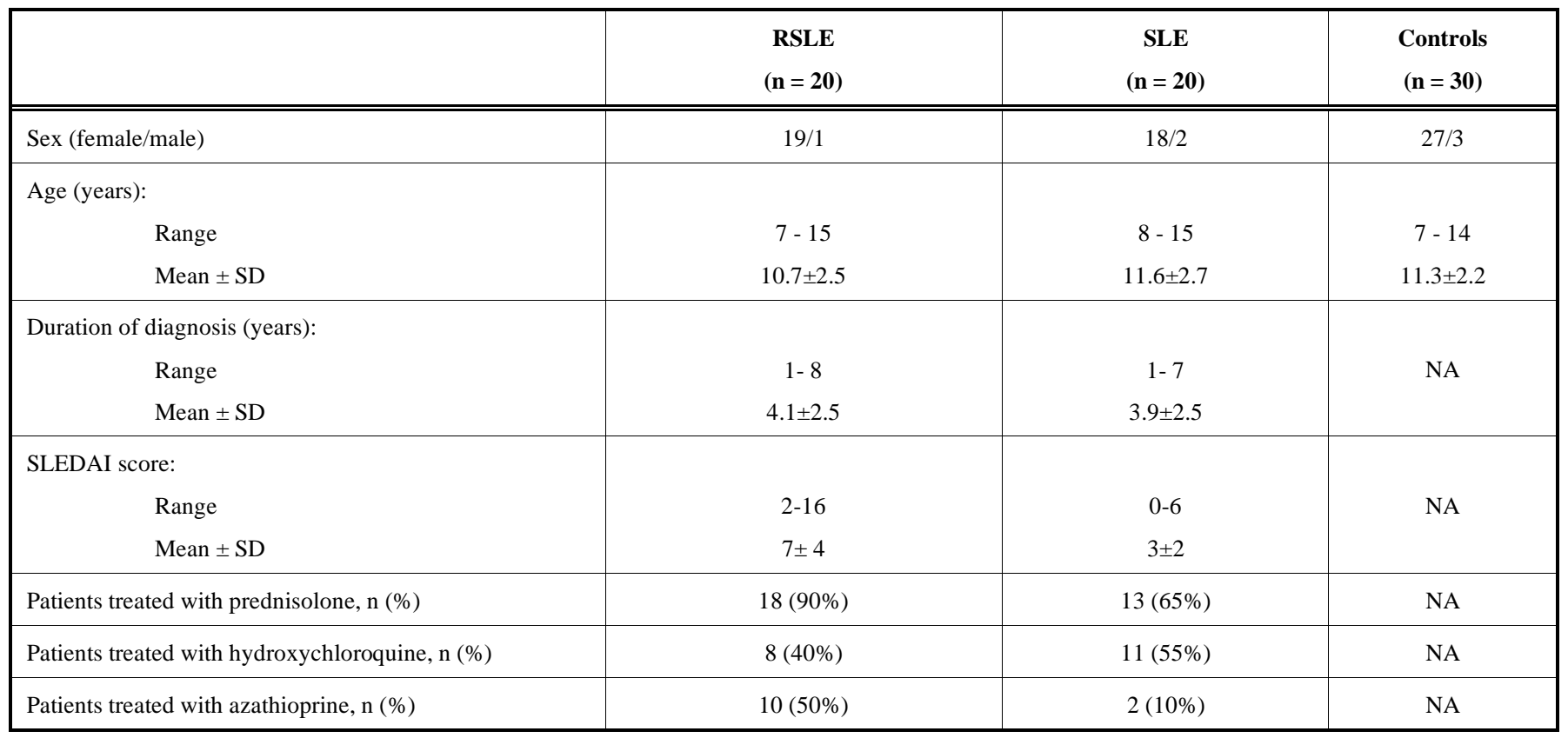

NA, not applicable. 
Table 2. Plasma OPN and IL-18 Concentrations of RSLE Patients, SLE Patients and Controls

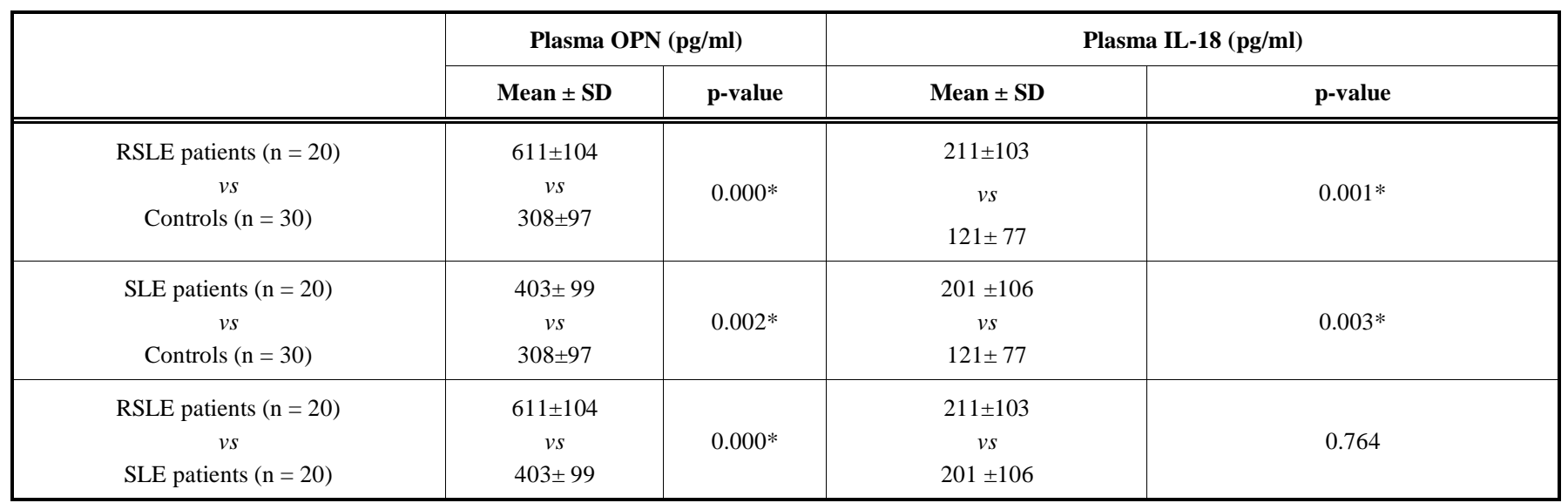

${ }^{*} \mathrm{p}<0.05$ is significant.

\section{Plasma Concentrations of OPN and IL-18}

Plasma OPN and IL-18 concentrations were significantly higher in RSLE and SLE patients than in controls. Plasma OPN concentrations were significantly higher in RSLE patients than in the SLE patients. On the other hand, there was no significant difference in IL-18 concentrations between RSLE and SLE patients (Table 2). Table 3 shows that there was a significant positive correlation between OPN concentration and SLEDAI score in combined SLE patient groups. Plasma IL-18 concentration was also found to correlate positively with SLEDAI in combined SLE patient groups and in both groups with or without renal disease. In addition, IL-18 showed a significant and positive correlation with OPN in RSLE patients (Table 4). Significant positive correlation was found between OPN concentration and 24 hour urinary protein in RSLE patients $(\mathrm{r}=0.461 ; \mathrm{p}=0.003)$. Also there was a significant positive correlation between IL-18 concentra- tion and 24 hour urinary protein in RSLE patients $(\mathrm{r}=0.370$; $\mathrm{p}=0.02$ ).

\section{DISCUSSION}

Previous studies have suggested that changes in Th1 and Th2 cytokines might be involved in the pathogenesis of autoimmune diseases [3]. SLE has been postulated to be an autoantibody-, immune complex- and Th2 cytokinemediated disease [25]. However, previous studies have reported different results for the correlation of the Th1/Th2 ratio with SLE disease activity. An in vitro study showed a positive and significant correlation of the ratio with SLEDAI using in vitro-stimulated peripheral blood mononuclear cells [3], while another reported a negative correlation of the ratio between IFN- $\gamma / \mathrm{IL}-10$-secreting cells and disease activity by enzyme-linked immunospot analysis of freshly isolated peripheral blood mononuclear cells [26]. Animal experiments

Table 3. Correlations of Plasma OPN and IL-18 Concentrations with SLEDAI in SLE Patients and RSLE Patients

\begin{tabular}{|ll|c|c|}
\hline Variable & r & p \\
\hline \hline OPN: & & & 0.83 \\
& SLE & 0.038 & 0.48 \\
& RSLE & 0.12 & $0.04 *$ \\
& SLE + RSLE & 0.34 & $0.02 *$ \\
IL-18: & & & $0.04 *$ \\
& SLE & 0.39 & $0.03 *$ \\
& RSLE & 0.35 & \\
SLE + RSLE & 0.36 & \\
\hline
\end{tabular}

*p $<0.05$ is significant.

Table 4. Correlations of Plasma Concentrations of OPN with IL-18 in SLE Patients and RSLE Patients

\begin{tabular}{|l|c|c|}
\hline & r & p \\
\hline \hline SLE & 0.07 & 0.68 \\
RSLE & 0.48 & $0.004^{*}$ \\
SLE + RSLE & 0.12 & 0.50 \\
\hline
\end{tabular}

* $p<0.05$ is significant. 
using autoimmune mice found that the ratio of Th1/Th2 cytokine mRNA expression of IL-2 and IL-4, IFN- $\gamma$ and IL-10 in polymorphonuclear neutrophils and peripheral blood mononuclear cells exhibited a reciprocal relationship with disease severity [27]. However, several clinical studies have indicated that elevation in Th1 cytokines, including IL-12 [5], TNF- $\alpha$ [28], and IFN- $\gamma$ [29], can mediate the inflammatory processes that lead to irreversible organ damage, such as renal failure in SLE [30]. The proinflammatory cytokine TNF- $\alpha$ was found to remain elevated throughout the course of disease [31], suggesting it has a significant role in the inflammatory process [30]. Previous studies have also demonstrated that elevated production of inflammatory mediators such as IL-18, nitric oxide, soluble thrombomodulin and soluble vascular cell adhesion molecule-1 is associated with renal disease in SLE patients, and the elevation of IL-18 was correlated with disease activity in SLE patients with renal impairment [32,33]. Altogether, these results suggest that Th1 predominance is important in SLE disease development.

OPN is one type of functional proteins in the extracellular matrix, which is characterized by a series of biological functions including cell adhesion, cytokine expression, cell migration, signal transduction, regulation of immunologic activity, and inhibition of cellular apoptosis. As a member of Th1 cytokines, OPN participates in inflammatory and immunologic reaction [17-22]. In this study we investigated the circulating level of OPN and its correlation with plasma IL-18 concentration and SLE disease activity.

The results of our present study indicate that the plasma concentration of cytokine OPN was significantly increased in SLE patients compared with controls, and correlated positively with SLEDAI. Similar finding was observed by Wong et al. [34] in adult patients with SLE. In addition, our results revealed that the plasma OPN concentration showed a significant positive correlation with proinflammatory cytokine IL-18 concentration in RSLE patients. Li et al. [35] also found that OPN and its mRNA expression are increased in peripheral blood mononuclear cells of SLE patients, while more obvious findings exist in lymphocyte too. Iizuka et al. [36] observed that over expression of OPN leads to enhanced $\mathrm{B}$ cell which causes increased anti-ds-DNA antibodies. The remarkably strong correlation between OPN and IL-18 in RSLE patients is interesting, especially since the RSLE patients were more medicated than the SLE group. There could be several possible reasons for this but the absence of such a correlation in the SLE group may suggest that cells in the kidney are either producing both cytokines or have an inductive relationship. In conjunction with the inflammatory activities of IL-18, such as the induction of Th1 cytokine IFN- $\gamma$ and activation of T cells, natural killer cells and cytotoxic T lymphocytes [37], OPN can enhance the Th1-mediated inflammatory process, activation of natural killer and $\mathrm{T}$ cells, and macrophage migration in the exacerbation of SLE. Acting together with other proinflammatory cytokines, including IL-1 and TNF- $\alpha$, OPN may be an important cytokine for initiating and perpetuating the Th1 immune response and renal derangement in SLE. The reported data showed that the change of OPN serum level is related to the activity of SLE, degree of renal damage and course of the disease itself [38]. In fact, OPN has been shown, at least partly, to account for SLE nephritis, probably through the predominance of the Thl-type response in both peripheral and renal tissues [21].

\section{CONCLUSION}

This study has provided new evidence for the association of the production of OPN with SLE disease development and renal derangement. In view of the above results, OPN may also act as a potential disease marker for the monitoring of SLE disease severity and therapeutic efficacy.

\section{REFERENCES}

[1] Kotzin BL. Systemic lupus erythematosus. Cell 1996; 85:303-6.

[2] Niadet P, Salmon R. Systemic Lupus Erythematosus. In: Avner ED, Harmon WE, Niadet P, Eds. Pediatric Nephrology. 5th ed. Philadelphia: Lippincott Williams \& Wilkins 2004; pp. 865-88.

[3] Viallard JF, Pellegrin JL, Ranchin V. Th1 (IL-2, interferon-gamma (IFN-gamma)) and Th2 (IL-10, IL-4) cytokine production by peripheral blood mononuclear cells (PBMC) from patients with systemic lupus erythematosus (SLE). Clin Exp Immunol 1999; 115: 189-95.

[4] Wong CK, Li EK, Lam CW. Elevation of plasma interleukin-18 concentration is correlated with disease activity in systemic lupus erythematosus. Rheumatology 2000; 39: 1078-81.

[5] Tokano Y, Morimoto S, Kaneko H. Levels of IL-12 in the sera of patients with systemic lupus erythematosus (SLE)-relation to Th1and Th2-derived cytokines. Clin Exp Immunol 1999; 116: 169-73.

[6] Wong CK, Ho CY, Lam CW. Elevation of proinflammatory cytokine (IL-18, IL-17, IL-12) and Th2 cytokine (IL-4) concentrations in patients with systemic lupus erythematosus. Lupus 2000; 9: 58993.

[7] Dinarello CA. Role of pro- and anti-inflammatory cytokines during inflammation: experimental and clinical findings. J Biol Regul Homeost Agents 1997; 11: 91-103.

[8] Akahoshi M, Nakashima H, Tanaka Y. Th1/Th2 balance of peripheral $\mathrm{T}$ helper cells in systemic lupus erythematosus. Arthritis Rheum 1999; 42: 1644-8.

[9] Chabas D, Baranzini SE, Mitchell D. The influence of the proinflammatory cytokine, osteopontin on autoimmune demyelinating disease. Science 2001; 294: 1731-5.

[10] Giachelli CM, Steitz S. Osteopontin: a versatile regulator of inflammation and biomineralization. Matrix Biol 2000; 19: 615-22.

[11] Wang KX, Denhardt DT. Osteopontin: role in immune regulation and stress responses. Cytokine Growth Factor Rev 2008 ; 19(5-6): $333-45$.

[12] Stromnes IM, Goverman JM. Osteopontin-induced survival of T cells. Nat Immunol 2007; 8(1): 19-20.

[13] Weber GF, Ashkar S, Cantor H. Receptor-ligand interaction between CD44 and osteopontin (Eta-1). Science 1996; 271: 509-12.

[14] Yokosaki Y, Higashikawa F. Osteopontin receptors and signal transduction. Nippon Rinsho 2005; 63(Suppl 10): 613-7.

[15] Liaw L, Birk DE, Hogan BL. Altered wound healing in mice lacking a functional osteopontin gene (spp1). J Clin Invest 1998; 101: 1468-78.

[16] Cao Z, Dai J, Guo Y. A novel functional motif of osteopontin for human lymphocyte migration and survival. Mol Immunol 2008; 45(14): 3683-92.

[17] Sato I, Yamamoto N, Uede T. Osteopontin is dispensable for protection against systemic infection. Int Immunopharmacol 2008; 8(10): 1441-8.

[18] Qin LX, Tang ZY. Recent progress in predictive biomarkers for metastatic recurrence of human hepatocellular carcinoma: a review of the literature. J Cancer Res Clin Oncol 2004; 130: 497-513.

[19] Altintaş A, Saruhan-Direskeneli G, Purisa S. The role of osteopontin: a shared pathway in the pathogenesis of multiple sclerosis and osteoporosis? J Neurol Sci 2009; 276(1-2): 41-4.

[20] Chiocchetti A, Indelicato M, Bensi T. High levels of osteopontin associated with polymorphisms in its gene is a risk factor for development of autoimmunity/lymphoproliferation. Blood 2004; 103 : 1376-82.

[21] Tang S, Lui SL, Lai KM. Pathogenesis of lupus nephritis. Nephrology 2005; 10(2): 174-9.

[22] Saeki Y. Osteopontin in Rrheumatoid Arthritis. Nippon Rinsho 2005; 63(Suppl 10): 627-31.

[23] Hochberg MC. Updating the American College of Rheumatology revised criteria for the classification of systemic lupus erythematosus. Arthritis Rheum 1997; 40: 1725. 
[24] Gladman DD, Ibañez D, Urowitz MB. Systemic lupus erythematosus disease activity index. J Rheumatol 2002; 29: 288-91.

[25] Mohan C, Adams S, Datta SK. Nucleosome: a major immunogen for pathogenic autoantibody-inducing T cells of lupus. J Exp Med 1993; 177: 1367-81.

[26] Hagiwara E, Gourley MF, Klinman DK. Disease severity in patients with systemic lupus erythematosus correlates with an increased ratio of interleukin-10: interferon-gamma-secreting cells in the peripheral blood. Arthritis Rheum 1996; 39: 379-85.

[27] Yu C, Sun K, Tsai C. Expression of Th1/Th2 cytokine mRNA in peritoneal exudative polymorphonuclear neutrophils and their effects on mononuclear cell Th1/Th2 cytokine production in MRLlpr/lpr mice. Immunology 1998; 95: 480-7.

[28] Davas EM, Tsirogianni A, Dantis PC. Serum IL-6, TNFalpha, p55 srTNFalpha, p75srTNFalpha, srIL-2alpha levels and disease activity in systemic lupus erythematosus. Clin Rheumatol 1999; 18: 1722

[29] Al-Janadi M, al-Balla S, Raziuddin S. Cytokine profile in systemic lupus erythematosus, rheumatoid arthritis, and other rheumatic diseases. J Clin Immunol 1993; 13: 58-67.

[30] Aringer M, Smolen JS. Tumour necrosis factor and other proinflammatory cytokines in systemic lupus erythematosus: a rationale for therapeutic intervention. Lupus 2004; 13: 344-7.

[31] Segal R, Bermas BL, Mozes E. Kinetics of cytokine production in experimental systemic lupus erythematosus: involvement of $\mathrm{T}$ helper cell 1/T helper cell 2-type cytokines in disease. J Immunol 1997; 158: 3009-16.

[32] Wong CK, Ho CY, Lam CW. Elevated production of interleukin18 is associated with renal disease in patients with systemic lupus erythematosus. Clin Exp Immunol 2002; 130: 345-51.

[33] Ho CY, Wong CK, Lam CW. Elevated plasma concentrations of nitric oxide, soluble thrombomodulin and soluble vascular cell adhesion molecule-1 in patients with systemic lupus erythematosus. Rheumatology 2003; 42: 117-22.

[34] Wong CK, Lit LW, Tam LS. Elevation of plasma osteopontin concentration is correlated with disease activity in patients with systemic lupus erythematosus. Rheumatology 2005; 44(5): 602-6.

[35] Li L, Yu XQ, Xu HS. OPN expression of peripheral blood mononuclear cells in systemic lupus erythematosus (SLE) and its clinical significance. Chin J Rheumatol 1999; 3: 84-6.

[36] Iizuka J, Katagiri Y, Sato M. Introduction of an osteopontin gene confers the increase in $\mathrm{B} 1$ cell population and the production of anti-DNA autoantibodies. Lab Invest 1998; 78: 1523-33.

[37] Dinarello CA. IL-18: A TH1-inducing, proinflammatory cytokine and new member of the IL-1 family. J Allergy Clin Immunol 1999; 103: 11-24.

[38] Lou B, Lv J, Zheng M. The relationship between osteopontin plasma concentration and disease activity in systemic lupus erythematosus. Chin J Dermatol 2006; 39: 320-1.

Received: April 21, 2009

Revised: May 24, 2009

Accepted: June 06, 2009

(C) Afify et al.; Licensee Bentham Open.

This is an open access article licensed under the terms of the Creative Commons Attribution Non-Commercial License (http://creativecommons.org/licenses/by-nc/3.0/) which permits unrestricted, non-commercial use, distribution and reproduction in any medium, provided the work is properly cited. 